\title{
Efficient fermentation of an improved synthetic grape must by enological and laboratory strains of Saccharomyces cerevisiae
}

Tiago Viana, Maria C Loureiro-Dias and Catarina Prista*

\begin{abstract}
Grape must or freshly pressed grape juice is a complex chemical matrix that impacts the efficiency of yeast fermentation. The composition of natural grape must (NGM) can be variable; thus, to ensure reproducibility, a synthetic grape must (SGM) with defined composition is commonly used. The aim of this work was to create conditions to advance the use of Saccharomyces cerevisiae laboratory strains for wine fermentation studies, considering previous results obtained for enological strains fermenting NGM under simulated winery conditions. We designed a new SGM formulation, ISA-SGM, by introducing specific modifications to a commonly used formulation, putting together previous reports. We added glucose and fructose in equal amounts $(125 \mathrm{~g} / \mathrm{l})$ and 50 parts per million $(\mathrm{ppm})$ sulfur dioxide $\left(\mathrm{SO}_{2}\right.$, corresponding to standard enological treatment), and we optimized the concentrations of malic acid (3 g/l), citric acid $(0.3 \mathrm{~g} / \mathrm{l})$, and tartaric acid (3 g/l). Using ISA-SGM, we obtained similar fermentative profiles for the wine strain ISA1000, the prototrophic strain S288C, and its auxotrophic derivative BY4741. In this case, the concentrations of supplements were optimized to $120 \mathrm{mg} / \mathrm{L}$-uracil, $80 \mathrm{mg} / \mathrm{l} \mathrm{L-methionine,} 400 \mathrm{mg} / \mathrm{l} \mathrm{L-leucine,} \mathrm{and} 100 \mathrm{mg} / \mathrm{l} \mathrm{L}$-histidine. All these strains tested in ISA-SGM presented a similar fermentative performance as ISA1000 in NGM. ISA-SGM formulation is a promising new tool to allow the use of the auxotrophic BY strains in the detailed assessment of the alcoholic fermentation process under simulated winery conditions, and it provides a foundation to extract relevant physiological conclusions in future research on enological yeast traits.
\end{abstract}

Keywords: Synthetic grape must; Natural grape must; Wine fermentation; BY auxotrophic mutant serie

\section{Introduction}

Saccharomyces cerevisiae plays a well-established and fundamental role in the complex process of winemaking. The prevalence of $S$. cerevisiae strains during grape must fermentation has been attributed to various factors, including strong fermentation capacity, high resistance to ethanol, and osmotolerance (Pretorius 2000). However, the physiology of $S$. cerevisiae also contributes to a chronic problem affecting the wine industry - the occurrence of stuck or sluggish (i.e. incomplete or delayed) fermentations that stop or slow down well before sugar exhaustion (Malherbe et al. 2007). Stuck fermentations are predominantly caused by the harmful effects of ethanol and other stress factors (Malherbe et al. 2007; Santos et al. 2008). Genes involved in tolerance to these factors could be

\footnotetext{
* Correspondence: cprista@isa.ulisboa.pt

Centro de Botânica Aplicada à Agricultura, Instituto Superior de Agronomia, Universidade de Lisboa, Lisboa, Portugal
}

targets for yeast genetic engineering to improve fermentation efficiency and to control the production of wine, at least as far as yeast performance is involved (Fleet 2008).

To elucidate genetic pathways relevant to winemaking, researchers have screened strains with deletion or overexpression of specific genes (Gómez-Pastor et al. 2010; López-Malo et al. 2012; Teixeira et al. 2009). However, the application of this strategy to wine strains has been impaired by the heterogeneity of industrial yeast genomes, which present frequently polyploidy or aneuploidy. These genomes often display heterozygosity, single nucleotide polymorphisms, strain-specific open reading frames, and localized variations in gene copy number (Borneman et al. 2011a,b; Bradbury et al. 2006; Pretorius 2000). This variability makes it difficult to construct single mutant libraries and to complete whole-genome screening in these strains. One strategy to understand the genetics of industrial wine yeasts is to develop genetically tractable versions 
of these commercial strains (Borneman et al. 2008; Engel and Cherry 2013; Walker et al. 2003, 2005). A single-gene deletion library is currently being developed in a haploid derivative of a wine yeast (Tran et al. 2010).

S. cerevisiae S288C is well-characterized and a commonly used laboratory strain that is the foundation of the single deletion mutant libraries (BY4741, BY4742, and BY4743) available at Euroscarf (Brachmann et al. 1998; Winzeler et al. 1999). These single-gene deletion and overexpression strains are a very powerful research tool for elucidating genetic pathways. However, S288C has been reported as a poorly fermenting strain when compared to industrial wine yeast strains (Harsch et al. 2010; Pizarro et al. 2007), even with amino acid supplementation (Harsch et al. 2010). The inferior fermentation ability is even more evident in the S288C-derived BY strains used in the systematic gene deletion project (Hanscho et al. 2012; Harsch et al. 2010). While it would be preferable to use wild strains for wine fermentation studies, mutants based on these strains are not yet available. S288C-derived BY strains are auxotrophic for several amino acids and uracil; thus, auxotrophic supplements must be added, appropriate concentrations of which have been determined by considering the composition of the final biomass and the type of energy metabolism (Pronk 2002). Auxotrophic supplementation is also necessary when using a complex medium, such as Yeast extract Peptone Dextrose (YPD) or grape must (Corbacho et al. 2011; Hanscho et al. 2012; Harsch et al. 2010). The optimal concentration of these supplements is still under debate. In addition to supplements for auxotrophy, other nutrients are required for the optimal growth and metabolic performances of S288C-derived BY strains. These nutrients include inositol, biotin, and mixtures of preferred amino acids (Çakar et al. 1999; Hanscho et al. 2012). The amounts and types of these supplements can trigger modifications of energy metabolism, protein expression, final biomass, survival capacity, and stress response (Brauer et al. 2008; Çakar et al. 1999; Görgens et al. 2005).

Grape must is a complex chemical matrix that depends on grape variety, ripeness stage, terroir characteristics, climate, and viticultural factors (Ribereau-Gayon et al. 2006). While transforming grape must into wine, yeast converts sugars to ethanol and produces various other compounds, which add new degrees of complexity and variability to the original matrix (Lambrechts and Pretorius 2000). The use of natural grape must (NGM) in the laboratory under simulated enological conditions is the best experimental approach and most precise way to study how wine yeast cope with stress conditions in the winery environment (Rossouw et al. 2012). However, considering the complexity and variability of NGM and the scientific requirement for reproducible data, researchers tend to use synthetic grape must (SGM) that has a known composition. Using SGM also allows researchers to avoid mutant phenotypes from being masked due to the presence of unknown substrates, especially when testing auxotrophic deletion mutants.

Several base formulations of SGM have been published that vary in the composition and concentration of their components (Bely et al. 1990; Ciani and Ferraro 1996; Riou et al. 1997). The most widely used SGM is MS300, a modified version of the synthetic grape juice first described by Bely et al. (1990). Several authors have introduced modifications to the MS300 composition. For example, researchers have replaced glucose with equimolar concentrations of glucose and fructose $(100$ and $150 \mathrm{~g} / \mathrm{l})$ as energy and carbon sources (Marullo et al. 2004), varied the amount of $\mathrm{SO}_{2}$ (Nardi et al. 2010), removed anaerobic growth factors (Gutiérrez et al. 2012), varied the concentrations of malic and citric acids (Albertin et al. 2011; Salmon and Barre 1998), and added tartaric acid (Albertin et al. 2011; Marullo et al. 2004). These modifications were introduced to make the SGM formulation more similar to that of NGM, and to optimize the media for optimal fermentation performance of laboratory strains (Harsch et al. 2010; Rossouw and Bauer 2009). Nonetheless, none of these formulations has been completely successful.

In the present work, we tested the ability of two closely related laboratory S. cerevisiae strains (S288C and BY4741) to ferment ISA-SGM under conditions similar to those found in wineries. To validate the use of the auxotrophic haploid strain BY4741 (Brachmann et al. 1998) as a model for wine yeast fermentation studies, the results obtained with this strain were compared with the behavior of an enological yeast strain under the same conditions. We combined suggestions made by some authors improving the formulation of a modified SGM, in which BY4741 can ferment with a comparable performance to that in NGM. This work aims to establish standard conditions for an efficient fermentation using BY4741 single mutation derivative strains to extract relevant physiological data on their fermentative performance in simulated wine fermentation studies.

\section{Materials and methods \\ Yeast strains}

The diploid S. cerevisiae S288C prototrophic strain and its isogenic auxotrophic haploid strain S. cerevisiae BY4741 (MATa his3D1 leu2DO met15D0; ura3DO (Brachmann et al. 1998)) were obtained from Euroscarf (http://web.unifrankfurt.de/fb15/mikro/euroscarf/index.html). In addition, an enological S. cerevisiae strain (ISA1000), isolated from a commercial active dry yeast starter (FERMIVIN ${ }^{\circ}$ ), was obtained from the Instituto Superior de Agronomia (ISA) yeast culture collection. Stock cultures were maintained at $4^{\circ} \mathrm{C}$ on YPD agar (1\% yeast extract, $2 \%$ peptone, $2 \%$ 
glucose, $2 \%$ agar). Yeast strains were stored at $-80^{\circ} \mathrm{C}$ in $15 \%$ glycerol and streaked onto YPD plates if necessary.

\section{Media and fermentation conditions Fermentative media}

First fermentations were carried out in synthetic grape must MS300 (Salmon and Barre 1998), which was modified as described by Rossouw and Bauer (2009) and designated as RB-SGM. Briefly, glucose and fructose (125 g/l each) were used as carbon and energy sources. The available nitrogen was $300 \mathrm{mg} / \mathrm{l}$, provided by $460 \mathrm{mg} / \mathrm{l} \mathrm{NH} \mathrm{NH}_{4} \mathrm{Cl}$ (corresponding to $120 \mathrm{mg} / \mathrm{l}$ of nitrogen) and by a mixture of 19 amino acids (corresponding to $180 \mathrm{mg} / \mathrm{l}$ of nitrogen). Ergosterol $(15 \mathrm{mg} / \mathrm{l})$ and sodium oleate $(5 \mathrm{mg} / \mathrm{l})$ in $1 \mathrm{ml}$ of Tween $80 /$ ethanol $(1: 1, \mathrm{v} / \mathrm{v})$ were added as anaerobic growth factors, and the $\mathrm{pH}$ was adjusted to 3.3 using $\mathrm{NaOH}$. Concentrated solutions of each compound were prepared, filtered through $0.22-\mu \mathrm{m}$ nitrocellulose membranes (Millipore filter, type GSWP), and added in adequate amounts before inoculation.

When indicated, RB-SGM was modified by varying the concentrations of citric, malic, and tartaric acids, to mimic the normal concentrations of these acids commonly found in must at grape maturity (Ribereau-Gayon et al. 2006). $\mathrm{SO}_{2}$ was adjusted to a final concentration of 50 parts per million (ppm) by adding potassium metabisulfite, as in standard enological treatments. When necessary, auxotrophic supplements were added at concentrations of $60 \mathrm{mg} / \mathrm{l} \mathrm{L}$-uracil, $200 \mathrm{mg} / \mathrm{l} \mathrm{L}$-leucine, $50 \mathrm{mg} / \mathrm{l} \mathrm{L}$-histidine, and $40 \mathrm{mg} / \mathrm{l} \mathrm{L-methionine}(1 \times)$ or twice these concentrations $(2 \times)$. The first values $(1 \times)$ approximately correspond to those recommended by Pronk (Pronk 2002), accounting for the biomass content and the expected final biomass obtained during wine fermentation (Viana et al. 2012). For comparison, fermentation of NGM from white grapes of the Arinto variety collected from the ISA vineyards, supplemented with $\mathrm{SO}_{2}(50 \mathrm{ppm})$, was carried out as described previously (Viana et al. 2012). Whenever necessary, the appropriate auxotrophic supplements indicated above were added to NGM.

\section{Fermentative conditions}

Yeast inoculation was standardized at $10^{6}$ cells $/ \mathrm{ml}$ in $80 \mathrm{ml}$ of liquid medium in $100 \mathrm{ml}$ Erlenmeyer flasks with a cotton cap (Viana et al. 2012). All fermentations were carried out at $25^{\circ} \mathrm{C}$ with very low orbital shaking (120 rpm) in a water bath (D-3162 Kottermann type 3047, West Germany). Fermentation progress was monitored by estimating the glucose concentration. After glucose exhaustion, samples were periodically collected for estimating fructose concentration in supernatants after removing the cells by centrifugation $(12,000 \times g, 3 \mathrm{~min}$, $4^{\circ} \mathrm{C}$ ). Fermentation was considered complete when the
Portuguese legal maximum limit for residual sugars in wine was reached $(\leq 2 \mathrm{~g} / \mathrm{l})$.

\section{Growth monitoring}

Yeast growth was monitored by measuring optical densities at $640 \mathrm{~nm}\left(\mathrm{OD}_{640 \mathrm{~nm}}\right)$ in an Ultrospec 2100 pro UV-visible (Amersham Biosciences ${ }^{\oplus}$ ) spectrophotometer. Growth data were analyzed using the DMFit software available on the Combase website (http://www.combase.cc/index.php/en/). Growth data were fit to the model proposed by Baranyi and Roberts (1994) to obtain lag times and specific growth rates of each fermentation assay. Viability was determined throughout fermentation by counting colony forming units (CFU) on YPD solid medium, after 2-3 days of incubation at $28^{\circ} \mathrm{C}$.

\section{Analytical techniques}

Dry biomass data were determined by filtering $1 \mathrm{ml}$ of cell suspension through preweighted Whatman membrane filters (pore diameter of $0.2 \mu \mathrm{m}$ ). The filters were rapidly washed with $10 \mathrm{ml}$ of distilled water, dried at $80^{\circ} \mathrm{C}$ for $24 \mathrm{~h}$, and weighed. Duplicate determinations varied by less than $5 \%$.

The glucose concentration was estimated with a rapid detection assay using a commercially available dipstick (Diabur-Test 5000; Boehringer, Mannheim, Germany) commonly used for measuring glucose in urine (range, 1-50 g/l) as previously described (Viana et al. 2012). Samples were periodically taken after glucose exhaustion, and the fructose concentration was estimated by using the Nelson-Somogyi method (Nelson 1944), as described previously (Fournier 2001). A calibration curve was created by correlating the $\mathrm{OD}_{620 \mathrm{~nm}}$ to the fructose concentration of standard solutions in the range of 0 to $2 \mathrm{~g} / \mathrm{l}$, using linear regression $\left(R^{2} \geq 0.99\right)$.

\section{Fermentation kinetics}

The duration of the lag phase was quantified as the time obtained by extrapolating the tangent at the exponential part of the growth curve, back to the inoculum level1 (Swinnen et al. 2004).

The time to the stationary phase was defined as the time necessary to reach the first of two equal OD values within a minimum interval of $5 \mathrm{~h}$. The end of glucose fermentation was defined as the time when glucose was exhausted $(0 \mathrm{~g} / \mathrm{l})$. The end of fermentation was defined as the time when the fructose concentration dropped below $2 \mathrm{~g} / \mathrm{l}$. These points were used to estimate the alcoholic fermentation times (in $\mathrm{h}$ ) and the time necessary to ferment glucose (AF1) and fructose (AF2), excluding the lag phase.

\section{Reproducibility of the results}

All experiments were performed at least 3 times. Mean values or results of typical experiments are presented, as indicated. 


\section{Results}

Strain S288C is a good laboratory model for white grape must fermentation

Recently, we characterized the fermentative performance of an enological yeast strain (S. cerevisiae ISA1000) during fermentation of NGM from the Portuguese white grape variety Arinto (Viana et al. 2012). In the present work, we studied the behavior of the laboratory haploid yeast strain S. cerevisiae S288C in NGM, comparing fermentation parameters of this strain with those obtained for the enological yeast S. cerevisiae ISA1000 under the same conditions. Figure 1 shows the growth curves and glucose consumptions for the two strains. Table 1 presents the corresponding macrokinetic parameters (i.e., lag-phase, doubling time, final OD, AF1 and AF2).

Both ISA1000 and S288C were able to ferment NGM efficiently up to the maximum legal limit for reducing sugars. Fermentation curves for the enological and laboratory strains showed that S288C grew more slowly during the exponential phase than ISA1000, with a specific growth rate of $(0.21 \pm 0.0079) / h$ compared $(0.31 \pm 0.0232) / \mathrm{h}$ to ISA1000. Nevertheless, S288C fermented glucose more rapidly than ISA1000; the time required for S288C to exhaust glucose (AF1) was $70 \mathrm{~h}$, whereas ISA1000 took $142 \mathrm{~h}$. The duration of fructose fermentation (AF2) was similar for both strains (150 and $166 \mathrm{~h}$, respectively) (Table 1). As expected, both strains fermented fructose at a slower rate than glucose.

\section{Improved SGM (ISA-SGM) leads to successful wine fermentations by S288C and ISA 1000}

To create a reliable SGM formulation for use in fermentations by laboratory strains, we modified a basal SGM formulation (RB-SGM) (Rossouw and Bauer 2009) by considering suggestions from Marullo et al. (2004) and Albertin et al. (2011), together with the data on the composition of NGMs from Ribereau-Gayon et al. (2006). The fermentative performance of S288C was evaluated in RB-SGM with the addition of $50 \mathrm{ppm} \mathrm{SO}_{2}$, anaerobic growth factors, and varying amounts and combinations of malic $(0,3$, and $6 \mathrm{~g} / \mathrm{l})$, citric $(0,0.3$, and $6 \mathrm{~g} / \mathrm{l})$, and tartaric acids $(0$ and $3 \mathrm{~g} / \mathrm{l})$. S288C fermenting basal RBSGM presented the same maximum growth rate as S288C fermenting NGM; however, it also had a significantly longer lag phase and a $49 \%$ reduction in the stationary phase biomass. S288C was not capable of completing the RBSGM fermentation, leaving $35 \mathrm{~g} / \mathrm{l}$ of residual glucose (Table 1 and Figure 2). Under these conditions, the fermentation was stuck. Among all of the tested formulations (results not shown), only the combination of $3 \mathrm{~g} / \mathrm{l}$ malic acid, $0.3 \mathrm{~g} / \mathrm{l}$ citric acid, and $3 \mathrm{~g} / \mathrm{l}$ tartaric acid allowed S288C to complete synthetic must fermentation up to must dryness (Figure 2). These values are close to those used by Wang et al. 2003.

The simultaneous introduction of small amounts of malic, citric, and tartaric acids along with anaerobic growth factors and $\mathrm{SO}_{2}$ led to a fermentative performance that was comparable to the fermentative performance of S288C in NGM (see values of AF1 and AF2 in Table 1). Therefore, we selected this formulation as our standard SGM, and designated it as ISA-SGM, for Instituto Superior de Agronomia-Synthetic Grape Must. Additional file 1 describes in detail the composition of ISA-SGM.

During S288C fermentation in both ISA-SGM and NGM, more than $50 \%$ of the yeast cells were viable (results not shown). This finding is similar to our previous result

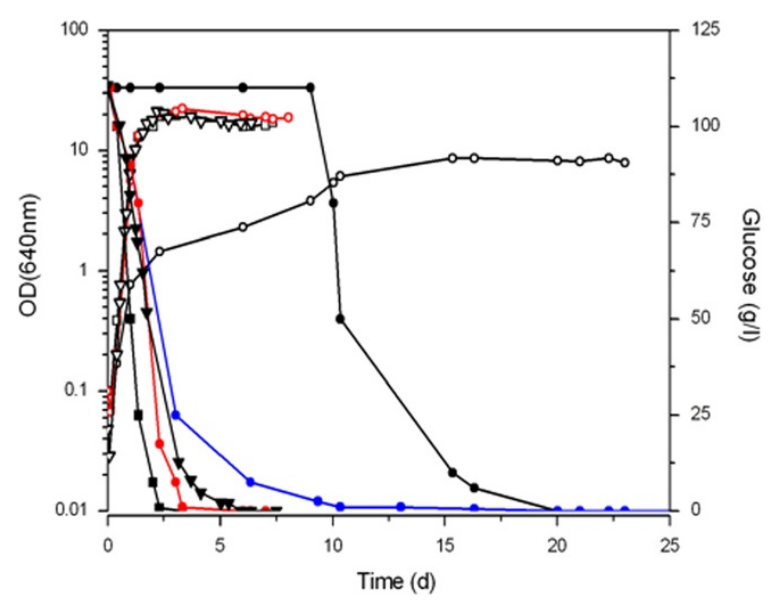

Figure 1 Fermentation performances of S. cerevisiae ISA1000, S288C and BY4741 strains in natural grape must (NGM). Open symbols indicate optical density for ISA1000 ( $\nabla$ ), S288C ( $\square$ ) and BY4741 (O and red circle symbol) strains, respectively. Glucose consumption is represented by closed symbols. Glucose was completely consumed by ISA1000 and S288C strains ( $\boldsymbol{\nabla}$ and $\mathbf{m}$ ), in NGM; for BY4741 strain glucose was completely consumed in NGM (•), in NGM with auxotrophic supplements (1x) (blue circle symbol) and in NGM with auxotrophic supplements (2x) (red circle symbol). All fermentations were performed at $25^{\circ} \mathrm{C}$. Representative results of one of three independent experiments are shown. OD, optical density. 
Table 1 Macrokinetic parameters for fermentations of NGM, RB-SGM and ISA-SGM by ISA1000, S288C and BY4741 strains

\begin{tabular}{|c|c|c|c|c|c|c|c|c|c|}
\hline Medium & Supp. addition ${ }^{a}$ & Strain & Lag-phase (h) & Doubling time (h) & Final OD & $\begin{array}{l}\text { Minimum } \\
\text { [Glucose] (g/L) }\end{array}$ & $A F 1^{b}(h)$ & $A F 2^{b}(h)$ & $\begin{array}{l}\text { Complete } \\
\text { fermentation }\end{array}$ \\
\hline \multirow[t]{5}{*}{ NGM } & - & ISA1000 & $<2$ & $2.4 \pm 0.18$ & $21.2 \pm 0.76$ & 0 & 142 & 166 & YES \\
\hline & - & S288C & $<2$ & $3.3 \pm 0.12$ & $20.4 \pm 1.87$ & 0 & 70 & 150 & YES \\
\hline & - & BY4741 & $4 \pm 0.67$ & $7.8 \pm 0.11$ & $8.8 \pm 0.43$ & 0 & 476 & 530 & YES \\
\hline & $1 x$ & BY4741 & $<2$ & $3.9 \pm 0.18$ & $15.3 \pm 0.83$ & 0 & 476 & 528 & YES \\
\hline & $2 x$ & BY4741 & $<2$ & $3.3 \pm 0.20$ & $19.6 \pm 1.42$ & 0 & 145 & 152 & YES \\
\hline RB-SGM & - & S288C & $40 \pm 0.84$ & $3.4 \pm 0.21$ & $10.4 \pm 0.88$ & 35 & $>570$ & $>570$ & NO \\
\hline \multirow[t]{5}{*}{ ISA-SGM } & - & ISA1000 & $3 \pm 0.75$ & $2.6 \pm 0.15$ & $23.4 \pm 0.64$ & 0 & 106 & 150 & YES \\
\hline & $2 x$ & ISA1000 & $4 \pm 0.74$ & $1.9 \pm 0.12$ & $22.6 \pm 0.74$ & 0 & 105 & 140 & YES \\
\hline & - & $\mathrm{S} 288 \mathrm{C}$ & $9 \pm 1.71$ & $3.4 \pm 0.21$ & $24.2 \pm 1.61$ & 0 & 159 & 183 & YES \\
\hline & $2 x$ & S288C & $8 \pm 0.71$ & $2.3 \pm 0.11$ & $23.3 \pm 0.97$ & 0 & 150 & 165 & YES \\
\hline & $2 x$ & BY4741 & $9 \pm 0.62$ & $3.6 \pm 0.26$ & $23.6 \pm 0.83$ & 0 & 159 & 300 & YES \\
\hline
\end{tabular}

Data represent the average of data from at least two independent cultures.

Standard deviation calculated from duplicate experiments ( \pm standard deviation).

${ }^{a}$ Concentration of auxotrophic supplements approximately corresponding to those described by Pronk (2002).

${ }^{b}$ AF1 (Alcoholic Fermentation for Glucose) and AF2 (Alcoholic Fermentation for Fructose) are the time for glucose and fructose fermentation excluding the lag-phase, respectively.

obtained for enological strain ISA1000 in NGM (Viana et al. 2012). We compared the fermentative performance of the commercial wine strain ISA1000 in ISA-SGM with our previous results of the ability of ISA1000 to ferment NGM (Viana et al. 2012). As shown in Figure 3, the growth curves were comparable in both types of media. The stationary phase was reached with a similar final biomass within a similar timeline (Table 1).

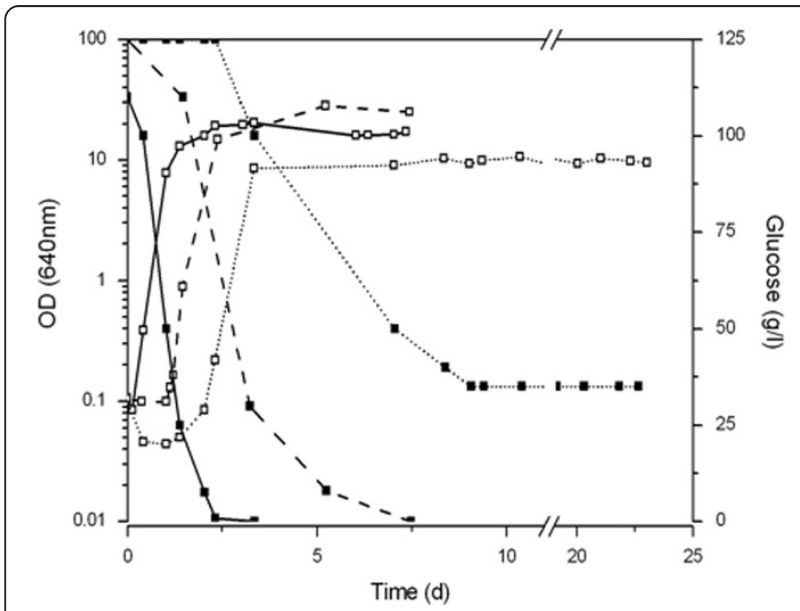

Figure 2 Fermentation performances of S. cerevisiae S288C strains in NGM, in RB-SGM and in ISA-SGM. Open squares ( $\square$ ) indicate optical density for S288C strain in NGM (solid line), in RB-SGM (dot line) and in ISA-SGM (dash line). Glucose ( $\mathbf{m}$ ) was completely consumed by S288C strain in NGM and in ISA-SGM, but in RB-SGM fermentation got stucked, lasting $35 \mathrm{~g} / \mathrm{l}$ of residual sugar in the medium. All fermentations were performed at $25^{\circ} \mathrm{C}$. Representative results of one of three independent experiments are shown. OD, optical density.
Although ISA-SGM contained $15 \mathrm{~g} / \mathrm{l}$ more of each sugar, the commercial strain was still able to ferment the sugars up to must dryness. Fermentation times were shorter for glucose in ISA-SGM (AF1 of 106 and $142 \mathrm{~h}$ for ISA-SGM and NGM, respectively) and were comparable for fructose (AF2 of 150 and $166 \mathrm{~h}$ for ISA-SGM and NGM, respectively) (Table 1). For ISA1000, we observed a cell viability of $50 \%$ at the end of ISA-SGM fermentation (results not

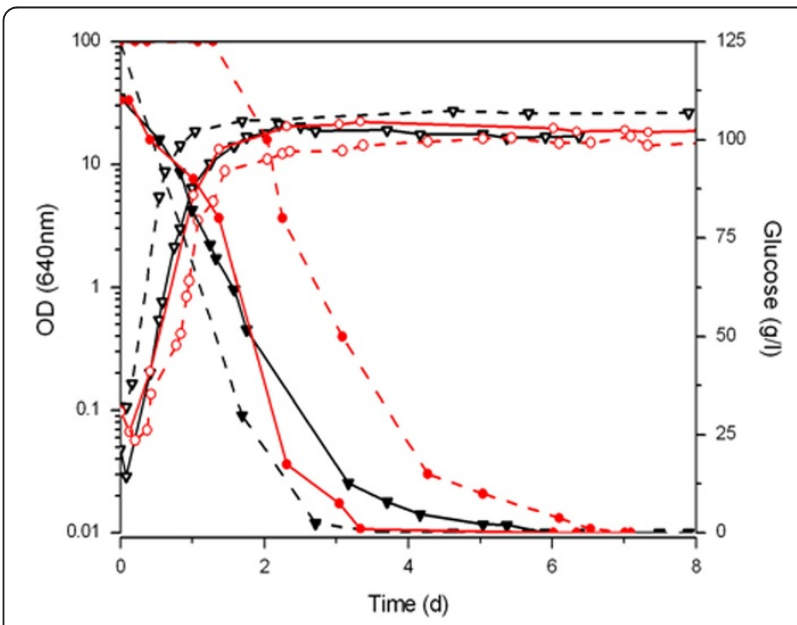

Figure 3 Fermentation performance of S. cerevisiae ISA 1000 and BY4741 strains in NGM and in ISA-SGM. Open symbols indicate optical density for ISA1000 strain ( $\nabla$ ) in NGM (black solid line) and in ISA-SGM (black dash line); for 2x auxotrophic supplemented NGM (red solid line) and ISA-SGM (red dash line) BY4741 growth is represented as (red circle symbol). Glucose was completely consumed for both strains ( $\boldsymbol{\nabla}$, red circle symbol) and in all media (solid and dash lines). All fermentations were performed at $25^{\circ} \mathrm{C}$. Representative results of one of three independent experiments are shown. OD, optical density. 
shown), consistent with our previous observations in NGM (Viana et al. 2012).

\section{Additional auxotrophic supplements improve fermentative performance of BY4741 in NGM and in ISA-SGM}

To validate the utility of the S288C-derived auxotrophic strain BY4741 and its single deletion mutant library available at Euroscarf, we performed fermentation assays with the BY4741 strain under the same conditions as described for ISA1000 and S288C. We also investigated the role of auxotrophic supplementation in NGM fermentation. When we compared the fermentative performances of S288C and BY4741 in NGM, the results confirmed a clear effect of the addition of extra supplementation on BY4741 behavior. BY4741 demonstrated the best fermentative performance with increased concentrations of auxotrophic supplements. Figure 1 and Table 1 show the growth kinetics of BY4741 in NGM supplemented with uracil, histidine, leucine, and methionine at $1 \times$ and $2 \times$ the previously recommended concentrations for anaerobic fermentation (Pronk 2002). Kinetics was monitored by $\mathrm{OD}_{640 \mathrm{~nm}}$ and by the sugar concentration, and was compared to S288C. Use of NGM without additional supplementation permitted complete must fermentation by BY4741; however, the BY4741 growth and sugar consumption parameters were severely affected. The lag-phase time was considerably increased, and the doubling time was decreased (specific growth rate for BY4741 was $(0.089 \pm$ $0.0012) / h$, much lower than that for S288C). The final $\mathrm{OD}_{640 \mathrm{~nm}}$ was only $43 \%$ of the $\mathrm{OD}_{640 \mathrm{~nm}}$ obtained for S288C under the same conditions.

However, the addition of $2 \times$ the auxotrophic supplements resulted in a specific growth rate $((0.21 \pm 0.013) / \mathrm{h})$ and final biomass $\left(\mathrm{OD}_{640 \mathrm{~nm}}\right.$ of 19.6) similar to those of S288C. With the auxotrophic supplements (2×), BY4741 took the same time to complete sugar fermentation (AF1 and AF2) as the enological strain S. cerevisiae ISA1000 (Table 1). As a control, strains ISA1000 and S288C were grown in the same medium: the inclusion of additional nitrogen sources did not disturbed the strains performance. Therefore, the slower fermentation of the auxotrophic strain was at least partially due to the auxotrophic mutations. Moreover, poor fermentation performance could be overcome by increasing the concentration of the appropriate supplements.

To validate the use of ISA-SGM in studies with the auxotrophic BY4741 strain, we compared the fermentative performances of this strain in ISA-SGM and in NGM. The concentrations of auxotrophic supplements were maintained at $2 \times$ the concentrations recommended for anaerobic fermentation (Pronk 2002). As we observed for S288C, fermentation of both sugars up to dryness was achieved by BY4741 in supplemented ISA-SGM (Figure 3 and Table 1). Additionally, when we compared BY4741 fermentations in ISA-SGM and NGM (both $2 \times$ supplemented), glucose was consumed in a similar time frame for both media (AF1) (Figure 3 and Table 1), and a similar $50 \%$ reduction in cell viability was observed (results not shown). However, some differences were still evident, as both the lag-phase and AF2 were significantly longer in ISA-SGM (Table 1).

\section{Discussion}

Natural grape must is a very complex and variable medium that has a great impact on the efficiency of yeast fermentation. The concentrations of sugar, nitrogen, and micronutrients change from season to season, and the composition of NGM depends on the grape variety, geographic and climate factors, viticulture practices, and the degree of fruit ripeness at harvest. The diversity of winemaking practices also contributes to the variability of grape must composition and fermentation conditions. Taken together, these factors make it difficult to compare fermentation performance between strains and studies. To achieve better experimental reproducibility, researchers frequently use SGMs with defined compositions when studying fermentation performance.

An increasing demand for new strains to optimize wine production has led to the development of yeast improvement programs (Giudici et al. 2005; Verstrepen et al. 2006). The selection of yeast strains with interesting features requires knowledge of yeast genetics and physiological diversity. To understand the fermentative behavior of yeast while fermenting grape must, the use of single deletion mutant collections fermenting defined SGMs may be a powerful approach. Yet, it is often difficult to extrapolate the behavior of laboratory strains under laboratory conditions to the behavior of commercial wine strains fermenting NGM under winery conditions. It is therefore mandatory a previous step of validation of both strain and must composition.

In this work, we aimed to test and validate the use of the parental S. cerevisiae S288C and its isogenic auxotrophic derivative BY4741 as laboratory models for wine fermentation studies. These strains presented fermentative performances similar with the known practical properties of the enological strain ISA1000 in NGM. We also designed a new SGM formulation (ISA-SGM), by adding glucose and fructose in equal amounts $(125 \mathrm{~g} / \mathrm{l})$ and 50 parts per million ( $\mathrm{ppm}$ ) sulfur dioxide by adding potassium metabisulfite (corresponding to standard enological treatment), and we optimized the concentrations of malic acid ( $3 \mathrm{~g} / \mathrm{l})$, citric acid $(0.3 \mathrm{~g} / \mathrm{l})$, and tartaric acid $(3 \mathrm{~g} / \mathrm{l})$, to mimic the normal concentrations of those acids commonly found in must at grape maturity (Ribereau-Gayon et al. 2006). We concluded that meaningful enological wine fermentation studies performed with S288C-derived laboratory strains could use ISA-SGM, as long as the proper concentrations of auxotrophic supplements are provided. When we com- 
pared the growth and fermentation properties of laboratory strain S288C in NGM with previous results obtained for the enological S. cerevisiae ISA1000 strain (Viana et al. 2012), both strains presented similar fermentative performances (Figure 1). It is likely that the NGM variability explains the discrepancies with previous results for the S288C strain, which had been described as an intrinsically poor fermenter (Harsch et al. 2010; Pizarro et al. 2007).

To avoid the variability of NGMs and the unpredictable behavior of laboratory strains during their fermentation, several SGMs have been designed (Harsch et al. 2010; Rossouw and Bauer 2009). However, as far as we know, none of these formulations has been completely successful. Moreover, most do not include $\mathrm{SO}_{2}$, which is common in standard enological practices. Therefore, we sought to develop a SGM to simulate the main stress factors found in NGM, including low vitamin concentration, high glucose and fructose concentrations, and the presence of weak acids. We mimicked winemaking practices by adding $\mathrm{SO}_{2}$ (final concentration of $50 \mathrm{ppm}$ ).

We determined that ISA-SGM was a good model of NGM, as measured by yeast growth and sugar consumption. Certainly, an important role is played by the increase of concentrations of malic, citric, and tartaric acids, which were closer to those commonly found in NGM. At lower concentrations of these acids, glucose was not exhausted and the fermentation became stuck. Only the combination of $3 \mathrm{~g} / \mathrm{l}$ malic acid, $0.3 \mathrm{~g} / \mathrm{l}$ citric acid, and $3 \mathrm{~g} / \mathrm{l}$ tartaric acid led to synthetic must fermentation up to dryness $(<2 \mathrm{~g} / \mathrm{l}$ residual sugar). Previous results may explain the effect of these weak acids. For example, it was shown that the presence of acetic acid causes an increase in glycolytic flux in yeast (Pampulha and Loureiro-Dias 2000). In the presence of weak acids, the Pma1 $\mathrm{H}^{+}$-ATPase mainly guarantees proton homeostasis. The additional $\mathrm{H}^{+}$outflow required for maintenance of the intracellular $\mathrm{pH}$ in the presence of weak acids dissipates extra ATP, decreasing cytosolic ATP pool. It has been reported that a decrease of the ATP levels in the cytoplasm stimulates the glycolytic flux (Larsson et al. 1997). It is conceivable that weak acids, which promote ATP consumption, contribute to increased sugar consumption rates and allow the SGM to reach dryness.

Because of its complex and variable nature, it is likely impossible to reproduce the composition of NGM completely. Despite the high performance in ISA-SGM, its composition does not match the complete composition of NGM. Particularly, it lacks the chemical precursors required for wine flavor (Styger et al. 2011; Swiegers and Pretorius 2005), which can affect yeast performance.

Using auxotrophic strains is convenient for detailed molecular studies of yeast fermentation, but their auxotrophy is a major drawback for the analysis of growth kinetics and stress effects. As a result, it can be difficult to translate results from the laboratory to an industrial setting (Pronk 2002). Additionally, the type of auxotrophic mutations, level of supplementation required, and particular growth medium used all have large effects on yeast growth kinetics. Often, the auxotrophic nutrient becomes limiting for growth (Bauer et al. 2003; Cohen and Engelberg 2007), inducing a physiological state that is different from that of cells whose growth is limited by standard biological nutrients, such as carbon, nitrogen, or phosphate (Brauer et al. 2008), or by metabolite toxicity. Despite these concerns, many of the genetic tools available for yeast have been constructed in auxotrophic strains.

While fermenting non-supplemented NGM, BY4741 fermented the must up to sugar exhaustion. Nevertheless, in non-supplemented NGM, BY4741 showed a moderate decrease in biomass levels and a significant increase in sugar fermentation times compared to the parental prototrophic S288C strain (Table 1). Experiments comparing the growth curves of the prototrophic S. cerevisiae S288C with the isogenic BY4741 auxotrophic mutant have reported similar results to ours for mineral and YPD media (Paciello et al. 2009).

Even NGM, a complex rich medium, did not fully compensate for the auxotrophic growth deficiencies of the BY4741 strain, which was unable to grow to its maximum biomass and accomplish an efficient wine fermentation in NGM. To overcome this deficiency, we hypothesized that the concentration of one or several essential supplements required for auxotrophy (uracil, leucine, histidine, and methionine) were present in concentrations below those required for optimized biomass production. Considering the expected maximum biomass reached during NGM fermentation (Viana et al. 2012) and the biomass content on each of the four supplements (Pronk 2002), we calculated minimum amounts to be added of $60 \mathrm{mg} / \mathrm{l}$ uracil, $40 \mathrm{mg} / \mathrm{l}$ methionine, $200 \mathrm{mg} / \mathrm{l}$ leucine, and $50 \mathrm{mg} / \mathrm{l} \mathrm{histi-}$ dine. These values are close to those recommended by Pronk (2002), considering that all of the supplements were used for biomass accumulation and not for further metabolism.

Assays were performed in NGM supplemented with the minimum and twice the amounts of these calculated concentrations. The minimum level of supplementation improved the fermentation parameters of BY4741. However, although the final biomass was closer to that of S288C in NGM, the sugar consumption by BY4741 was still significantly slower than that of S288C (Table 1, Figure 1). Supplementing NGM with the highest concentrations of auxotrophic supplements $(120 \mathrm{mg} / \mathrm{l}$ uracil, $80 \mathrm{mg} / \mathrm{l}$ methionine, $400 \mathrm{mg} / \mathrm{l}$ leucine, and $100 \mathrm{mg} / \mathrm{l}$ histidine) further increased the final biomass of BY4741 to levels of the prototrophic parental strain S288C and the enological 
commercial strain ISA1000 fermenting NGM without auxotrophic supplements (Table 1, Figure 1). In addition, the AF1 and AF2 fermentation times in the $2 \times$ supplemented NGM were reduced to approximately $30 \%$ of the time required to ferment NGM without extra supplementation. These fermentation times were similar to those presented by S288C and ISA1000 fermenting NGM without extra supplementation.

In conclusion, we have developed a synthetic grape must, ISA-SGM, in which a prototrophic laboratory strain and an auxotrophic strain (appropriately supplemented) present fermentative profiles similar to that of a commercial wine strain in natural grape must. ISA-SGM can be used as a new tool for BY mutants in the detailed assessment of the alcoholic fermentation process under conditions close to those found in wineries. This new formulation may provide a sound foundation to extract relevant physiological conclusions on enological molecular yeast traits.

\section{Additional file}

Additional file 1: Chemical composition of Instituto Superior de Agronomia - Synthetic Grape Must (ISA-SGM). pH was adjusted to 3.3 using $\mathrm{NaOH}$. Concentrated solutions of each compound were prepared, filtered through $0.22-\mu \mathrm{m}$ nitrocellulose membranes (Millipore filter, type GSWP), and added in adequate amounts before inoculation.

\section{Competing interests}

The authors declare that the research was conducted in the absence of any commercial or financial relationships that could be construed as potential conflicts of interest.

\section{Acknowledgements}

This work was supported by Fundação para a Ciência e a Tecnologia (PTDC/ AGR-ALI/112795/2009, SFRH/BD/65236/2009, SFRH/BPD/85143/2012 and a contract FCT Ciência 2007).

Received: 10 February 2014 Accepted: 13 February 2014 Published online: 01 April 2014

\section{References}

Albertin W, Marullo P, Aigle M, Dillmann C, de Vienne D, Bely M, Sicard D (2011) Population size drives industrial Saccharomyces cerevisiae alcoholic fermentation and is under genetic control. Appl Environ Microbiol 77:2772-2784. doi:10.1128/ AEM.02547-10

Baranyi J, Roberts TA (1994) A dynamic approach to predicting bacterial growth in food. Int J Food Microbiol 23:277-294. doi:10.1016/0168-1605(94)90157-0

Bauer BE, Rossington D, Mollapour M, Mamnun Y, Kuchler K, Piper PW (2003) Weak organic acid stress inhibits aromatic amino acid uptake by yeast, causing a strong influence of amino acid auxotrophies on the phenotypes of membrane transporter mutants. Eur J Biochem 270:3189-3195. doi:10.1046/ j.1432-1033.2003.03701.x

Bely M, Sablayrolles J-M, Barre P (1990) Automatic detection of assimilable nitrogen deficiencies during alcoholic fermentation in oenological conditions. J Ferment Bioeng 70:246-252. doi:10.1016/0922-338X(90)90057-4

Borneman AR, Forgan AH, Pretorius IS, Chambers PJ (2008) Comparative genome analysis of a Saccharomyces cerevisiae wine strain. FEMS Yeast Res 8:1185-1195. doi:10.1111/j.1567-1364.2008.00434.x

Borneman AR, Desany BA, Riches D, Affourtit JP, Forgan AH, Pretorius IS, Egholm M, Chambers PJ (2011a) The genome sequence of the wine yeast VIN7 reveals an allotriploid hybrid genome with Saccharomyces cerevisiae and Saccharomyces kudriavzevii origins. FEMS Yeast Res 12:88-96. doi:10.1111/j.1567-1364.2011.00773.x
Borneman AR, Desany BA, Riches D, Affourtit JP, Forgan AH, Pretorius IS, Egholm M, Chambers PJ (2011b) Whole-genome comparison reveals novel genetic elements that characterize the genome of industrial strains of Saccharomyces cerevisiae. PLoS Genet 7:e1001287. doi:10.1371/journal.pgen.1001287

Brachmann CB, Davies A, Cost GJ, Caputo E, Li J, Hieter P, Boeke JD (1998) Designer deletion strains derived from Saccharomyces cerevisiae S288C: a useful set of strains and plasmids for PCR-mediated gene disruption and other applications. Yeast 14:115-132. doi:10.1002/(SICI)1097-0061(19980130) 14:2<115::AID-YEA204>3.0.CO;2-2

Bradbury J, Richards K, Niederer H, Lee S, Rod Dunbar P, Gardner R (2006) A homozygous diploid subset of commercial wine yeast strains. Antonie van Leeuwenhoek 89:27-37-37. doi:10.1007/s10482-005-9006-1

Brauer MJ, Huttenhower C, Airoldi EM, Rosenstein R, Matese JC, Gresham D, Boer VM, Troyanskaya OG, Botstein D (2008) Coordination of growth rate, cell cycle, stress response, and metabolic activity in yeast. Mol Biol Cell 19:352-367. doi:10.1091/mbc.E07-08-0779

Çakar ZP, Sauer U, Bailey J (1999) Metabolic engineering of yeast: the perils of auxotrophic hosts. Biotechnol Lett 21:611-616. doi:10.1023/A:1005576004215

Ciani M, Ferraro L (1996) Enhanced glycerol content in wines made with immobilized Candida stellata cells. Appl Environ Microb 62:128-132

Cohen R, Engelberg D (2007) Commonly used Saccharomyces cerevisiae strains (e.g. BY4741, W303) are growth sensitive on synthetic complete medium due to poor leucine uptake. FEMS Microbiol Lett 273:239-243. doi:10.1111/j.15746968.2007.00798.x

Corbacho I, Teixidó F, Velázquez R, Hernández LM, Olivero I (2011) Standard YPD, even supplemented with extra nutrients, does not always compensate growth defects of Saccharomyces cerevisiae auxotrophic strains. Antonie van Leeuwenhoek 99:591-600. doi:10.1007/s10482-010-9530-5

Engel SR, Cherry JM (2013) The New Modern Era of Yeast Genomics: Community Sequencing and the Resulting Annotation of Multiple Saccharomyces cerevisiae Strains at the Saccharomyces Genome Database. Database, Oxford. doi:10.1093/database/bat012

Fleet GH (2008) Wine yeasts for the future. FEMS Yeast Res 8:979-995. doi:10.1111/j.1567-1364.2008.00427.x

Fournier E (2001) Colorimetric Quantification of Carbohydrates. In: Wrolstad (ed) Current Protocols in Food Analytical Chemistry. John Wiley \& Sons, Inc, Alberta, Canada, E1.1.1-E1.1.8. doi: 10.1002/0471142913.fae0101s00

Giudici P, Solieri L, Pulvirenti A, Cassanelli S (2005) Strategies and perspectives for genetic improvement of wine yeasts. Appl Microbiol Biotechnol 66:622-628. doi:10.1007/s00253-004-1784-2

Gómez-Pastor R, Pérez-Torrado R, Cabiscol E, Ros J, Matallana E (2010) Reduction of oxidative cellular damage by overexpression of the thioredoxin TRX2 gene improves yield and quality of wine yeast dry active biomass. Microb Cell Fact 9:9-23. doi:10.1186/1475-2859-9-9

Görgens J, Zyl W, Knoetze J, Hahn-Hägerdal B (2005) Amino acid supplementation improves heterologous protein production by Saccharomyces cerevisiae in defined medium. Appl Microbiol Biotechnol 67:684-691. doi:10.1007/ s00253-004-1803-3

Gutiérrez A, Chiva R, Sancho M, Beltran G, Arroyo-López FN, Guillamón JM (2012) Nitrogen requirements of commercial wine yeast strains during fermentation of a synthetic grape must. Food Microbiol 31:25-32. doi:10.1016/j.fm.2012.02.012

Hanscho M, Ruckerbauer DE, Chauhan N, Hofbauer HF, Krahulec S, Nidetzky B, Kohlwein SD, Zanghellini J, Natter K (2012) Nutritional requirements of the BY series of Saccharomyces cerevisiae strains for optimum growth. FEMS Yeast Res 12:796-808. doi:10.1111/j.1567-1364.2012.00830.x

Harsch MJ, Lee SA, Goddard MR, Gardner RC (2010) Optimized fermentation of grape juice by laboratory strains of Saccharomyces cerevisiae. FEMS Yeast Res 10:72-82. doi:10.1111/j.1567-1364.2009.00580.x

Lambrechts MG, Pretorius IS (2000) Yeast and its importance to wine aroma - a review. S Afric J Enol Vitic 21:97-129

Larsson C, Nilsson A, Blomberg A, Gustafsson L (1997) Glycolytic flux is conditionally correlated with ATP concentration in Saccharomyces cerevisiae: a chemostat study under carbon- or nitrogen-limiting conditions. J Bacteriol 179:7243-7250

López-Malo M, Chiva R, Rozès N, Guillamón JM (2012) Phenotypic analysis of mutant and overexpressing strains of lipid metabolism genes in Saccharomyces cerevisiae: Implication in growth at low temperatures. Int J Food Microbiol 162:26-36, http://dx.doi.org/10.1016/j.ijfoodmicro.2012.12.020

Malherbe S, Bauer FF, Toit MD (2007) Understanding problem fermentations - a review. S Afric J Enol Vitic 28:169-186

Marullo P, Bely M, Masneuf-Pomarede I, Aigle M, Dubourdieu D (2004) Inheritable nature of enological quantitative traits is demonstrated by meiotic 
segregation of industrial wine yeast strains. FEMS Yeast Res 4:711-719. doi:10.1016/j.femsyr.2004.01.006

Nardi T, Corich V, Giacomini A, Blondin B (2010) A sulphite-inducible form of the sulphite efflux gene SSU1 in a Saccharomyces cerevisiae wine yeast. Microbiology 156:1686-1696. doi:10.1099/mic.0.036723-0

Nelson N (1944) A photometric adaptation of the Somogyi method for the determination of glucose. J Biol Chem 153:375-379

Paciello L, de Alteriis E, Mazzoni C, Palermo V, Zueco J, Parascandola P (2009) Performance of the auxotrophic Saccharomyces cerevisiae BY4741 as host for the production of IL-1beta in aerated fed-batch reactor: role of ACA supplementation, strain viability, and maintenance energy. Microb Cell Fact 8:70-83. doi:10.1186/ 1475-2859-8-70

Pampulha ME, Loureiro-Dias MC (2000) Energetics of the effect of acetic acid on growth of Saccharomyces cerevisiae. FEMS Microbiol Lett 184:69-72. doi:10.1111/j.1574-6968.2000.tb08992.x

Pizarro F, Vargas FA, Agosin E (2007) A systems biology perspective of wine fermentations. Yeast 24:977-991. doi:10.1002/yea.1545

Pretorius IS (2000) Tailoring wine yeast for the new millennium: novel approaches to the ancient art of winemaking. Yeast 16:675-729. doi:10.1002/ 1097-0061(20000615)16:8<675::AID-YEA585>3.0.CO;2-B

Pronk JT (2002) Auxotrophic yeast strains in fundamental and applied research. Appl Environ Microb 68:2095-2100

Ribereau-Gayon P, Dubourdieu D, Donèche B, Lonvaud A (2006) The chemistry of wine. In: Ribereau-Gayon P et al. (ed) Handbook of enology, the microbiology of wine and vinifications. John Wiley \& Sons, Ltd, Chichester, UK, pp 1-228

Riou C, Nicaud J-M, Barre P, Gaillardin C (1997) Stationary-phase gene expression in Saccharomyces cerevisiae during wine fermentation. Yeast 13:903-915. doi:10.1002/(SICI)1097-0061(199708)13:10<903:.AID-YEA145>3.0.CO;2-1

Rossouw D, Bauer FF (2009) Comparing the transcriptomes of wine yeast strains: toward understanding the interaction between environment and transcriptome during fermentation. Appl Microbiol Biotechnol 84:937-954. doi:10.1007/s00253-009-2204-4

Rossouw D, Jolly N, Jacobson D, Bauer FF (2012) The effect of scale on gene expression: commercial versus laboratory wine fermentations. Appl Microbiol Biotechnol 93:1207-1219. doi:10.1007/s00253-011-3564-0

Salmon J-M, Barre P (1998) Improvement of nitrogen assimilation and fermentation kinetics under enological conditions by derepression of alternative nitrogen-assimilatory pathways in an industrial Saccharomyces cerevisiae strain. Appl Environ Microbiol 64:3831-3837

Santos J, Sousa MJ, Cardoso H, Inácio J, Silva S, Spencer-Martins I, Leão C (2008) Ethanol tolerance of sugar transport, and the rectification of stuck wine fermentations. Microbiology 154:422-430. doi:10.1099/mic.0.2007/011445-0

Styger G, Prior B, Bauer F (2011) Wine flavor and aroma. J Ind Microbiol Biotechnol 38:1145-1159. doi:10.1007/s10295-011-1018-4

Swiegers JH, Pretorius IS (2005) Yeast modulation of wine flavor. In: Laskin Al, Gadd GM, Bennett JW (ed) Advances in applied microbiology. Academic Press, United States. pp 131-175

Swinnen IAM, Bernaerts K, Dens EJJ, Geeraerd AH, Van Impe JF (2004) Predictive modelling of the microbial lag phase: a review. Int J Food Microbiol 94:137-159. doi:10.1016/j.jijfoodmicro.2004.01.006

Teixeira MC, Raposo LR, Mira NP, Lourenco AB, Sa-Correia I (2009) Genome-wide identification of Saccharomyces cerevisiae genes required for maximal tolerance to ethanol. Appl Environ Microb 75:5761-5772. doi:10.1128/AEM.00845-09

Tran T, Forgan AH, Bartowsky EJ, Borneman A (2010) Construction of a wine yeast genome deletion library. 12th International Conference on Culture Collections, Santa Catarina, Brazil. http://www.wfcc.info/iccc12/abstract/ ab_tina.pdf. Accessed 12 December 2013

Verstrepen K, Chambers P, Pretorius I (2006) The development of superior yeast strains for the food and beverage industries: challenges, opportunities and potential benefits. In: Querol A, Fleet G (eds) Yeasts in Food and Beverages. Springer, Berlin Heidelberg, pp 399-444

Viana T, Loureiro-Dias MC, Loureiro V, Prista C (2012) Peculiar $\mathrm{H}^{+}$homeostasis of Saccharomyces cerevisiae during the late stages of wine fermentation. Appl Environ Microbiol 78:6302-6308. doi:10.1128/AEM.01355-12

Walker ME, Gardner JM, Vystavelova A, McBryde C, de Barros LM, Jiranek V (2003) Application of the reuseable, KanMX selectable marker to industrial yeast: construction and evaluation of heterothallic wine strains of Saccharomyces cerevisiae, possessing minimal foreign DNA sequences. FEMS Yeast Res 4:339-347. doi:10.1016/S1567-1356(03)00161-2

Walker M, Vystavelova A, Pedler S, Eglinton J, Jiranek V (2005) PCR-based gene disruption and recombinatory marker excision to produce modified industrial
Saccharomyces cerevisiae without added sequences. J Microbiol Meth 63:193-204. doi:10.1016/j.mimet.2005.03.015

Wang XD, Bohlscheid JC, Edwards CG (2003) Fermentative activity and production of volatile compounds by Saccharomyces grown in synthetic grape juice media deficient in assimilable nitrogen and/or pantothenic acid. J Appl Microbiol 94:349-359

Winzeler EA, Shoemaker DD, Astromoff A, Liang H, Anderson K, Andre B, Bangham R, Benito R, Boeke JD, Bussey H, Chu AM, Connelly C, Davis K, Dietrich F, Dow SW, El Bakkoury M, Foury F, Friend SH, Gentalen E, Giaever G, Hegemann JH, Jones T, Laub M, Liao H, Liebundguth N, Lockhart DJ, Lucau-Danila A, Lussier M, M'Rabet N, Menard P et al (1999) Functional characterization of the S. cerevisiae genome by gene deletion and parallel analysis. Science 285:901-906

\section{doi:10.1186/s13568-014-0016-0}

Cite this article as: Viana et al:: Efficient fermentation of an improved synthetic grape must by enological and laboratory strains of Saccharomyces cerevisiae. AMB Express 2014 4:16.

\section{Submit your manuscript to a SpringerOpen ${ }^{\circ}$ journal and benefit from:}

- Convenient online submission

- Rigorous peer review

- Immediate publication on acceptance

- Open access: articles freely available online

- High visibility within the field

- Retaining the copyright to your article

Submit your next manuscript at $>$ springeropen.com 